\title{
Reduced Hospital Stay in Influenza Patients after Mass Vaccination during the 2009 Influenza Pandemic in Norway
}

Kristin Greve-Isdahl Mohn ${ }^{1,2 *}$, Birger N Lærum ${ }^{1}$, Steinar Skrede ${ }^{1,3}$, Rebecca J Cox ${ }^{2,4}$, Anne Ma Dyrhol-Riise ${ }^{1,3}$, Hans Erling Simonsen ${ }^{1}$, Nina Langeland ${ }^{1,3}$, Jörg Aßmus ${ }^{5}$, Per-Espen Akselsen ${ }^{1,4}$, Haakon Sjursen ${ }^{1,3}$ and Ingrid Smith ${ }^{1,4}$

${ }^{1}$ Infectious Diseases Unit, Department of Internal Medicine, Haukeland University Hospital, N-5021 Bergen, Norway

${ }^{2}$ Influenza Centre and Jebsen Centre for Influenza vaccine research, Department of Clinical Sciences, University of Bergen, N-5021 Bergen, Norway

${ }^{3}$ Department of Clinical Sciences, University of Bergen, N-5021 Bergen, Norway

${ }^{4}$ Department of Research and Development, Centre of Infection Control, Haukeland University Hospital, N-5021 Bergen, Norway

${ }^{5}$ Department of Research and Development, Centre for Clinical Research, Haukeland University Hospital, N-5021 Bergen, Norway

\begin{abstract}
Background: Norway had a preorder of pandemic vaccine, when the pandemic influenza A (H1N1)pdm09 in 2009 was declared. Mass vaccination occurred 1-3 weeks prior to the peak of the pandemic. Emergency plans were in place, but the predicted severe numbers of hospitalizations did not occur.

Objective: To study the epidemiology and clinical presentation of adult patients hospitalized with Influenza $\mathrm{A}(\mathrm{H} 1 \mathrm{~N} 1) p d m 09$, and to evaluate the impact of vaccination on the course of the pandemic at a tertiary hospital.

Methods: The low dose oil-in-water adjuvanted vaccine was used to vaccinate healthcare workers (HCWs) and at risk patients groups, and vaccination rates were recorded for the community and the hospital. Demographic and clinical information was obtained for 129 patients (>15 years), hospitalized with influenza A (H1N1)pdm09 between August 2009-January 2010. A confirmed case of influenza A (H1N1)pdm09 was defined as meeting a clinical case definition and/or laboratory confirmed disease (rt-PCR or serology). Hospital stay of more than 2 days was defined as a sign of severe illness.

Results: $1 / 3$ of at risk patients in the community and $>90 \%$ of frontline HCWs at the hospital were vaccinated The median length of hospital stay of infected patients was significantly reduced 7 days after the onset of mass vaccination $(p=0.029)$. There was a predominance of female and moderately obese (BMI 25-30) patients. Infiltration on chest $X$-ray upon admission was significantly associated with a hospital stay of $>2$ days $(p=0.001)$.

Conclusion: Mass vaccination of frontline $\mathrm{HCWs}$ at the hospital and at risk patients in the community contributed to the observed significant reduction in hospital stay of patients infected with influenza. Almost no absenteeism enabled staff confidence and the ability for quick and safe patient turnover. This study highlights the importance of early influenza vaccination, to protect the high-risk patients and the integrity of the healthcare system.
\end{abstract}

Keywords: Influenza A(H1N1)pdm09; Pandemic, Mass vaccination; Healthcare worker; Hospital, Risk patients, Community, Epidemiology

\section{Introduction}

The emergence of a new influenza pandemic had long been anticipated since the 1968 Hong Kong Influenza H3N2 pandemic. The focus of pandemic preparedness had in later years been on the zoonosis caused by the highly pathogenic avian influenza virus (H5N1), and on increased surveillance of man and poultry, particularly in South-East Asia. Hence the world was taken somewhat by surprise when Mexico in April 2009 alerted the world to a novel influenza A virus of swine origin, subsequently referred to as Influenza A(H1N1)pdm09, heralding the advent of the first influenza pandemic for 4 decades [1,2].

Influenza vaccination remains the most effective prophylactic measure to prevent infection and limit viral spread in the general population. With limited vaccine-manufacturing capacity, a clear global and national prioritized vaccination strategy was developed. The WHO Strategic Advisory Group of Experts (SAGE) on Immunization recommended two high priority groups for vaccination: frontline health care workers (HCW) and high risk populations, including pregnant women, individuals with $\mathrm{BMI}>40 \mathrm{~kg} / \mathrm{m}^{2}$, and people $<65$ years with asthma/ chronic obstructive pulmonary disease (COPD), diabetes, chronic heart, - kidney, - hepatic or neurologic disease or immunocompromised individuals. Vaccination of HCWs was recommended to ensure the integrity of the health care system, reduce absenteeism and prevent spread of the virus in the hospital [3-6].
Norway, with a population of 4.9 million people [7], was fortunate to be well informed of the on-going pandemic [8,9-13] Preparedness plans were in place. Due to a pre order, the pandemic vaccine became rapidly available 1-3 weeks prior to the peak pandemic activity. Norway was one of the European countries with the highest vaccination coverage (45\%). Haukeland University Hospital (HUH) provides specialized healthcare for approximately 260000 inhabitants in Bergen, and is a tertiary hospital for Western-Norway, serving a population of 1027 $000[7,14]$. Vaccination among HCWs was voluntary, and willingness to become vaccinated increased with serious patient reports, and the death of a patient with no known risk factor at our hospital on October $18^{\text {th }}$ (week 42). Immunization of frontline HCWs and the population at risk in Bergen commenced on October $21^{\text {st }} 2009$ (week 43), coinciding

*Corresponding author: Kristin Greve-Isdahl Mohn, Infectious Diseases Unit, Department of Internal Medicine, Haukeland University Hospital, N-5021 Bergen, Norway. Influenza Center, Department of Clinical Sciences, University of Bergen, N-5021 Bergen, Norway, Tel: +47 559758 00/+47 977707 55; E-mail: kristin.mohn@k2.uib.no

Received June 26, 2013; Accepted August 20, 2013; Published August 23, 2013 Citation: Mohn KGI, Lærum BN, Skrede S, Cox RJ, Dyrhol-Riise AM, et al. (2013) Reduced Hospital Stay in Influenza Patients after Mass Vaccination during the 2009 Influenza Pandemic in Norway. J Vaccines Vaccin 4: 197. doi: 10.4172/2157. 7560.1000197

Copyright: (c) 2013 Mohn KGI, et al. This is an open-access article distributed under the terms of the Creative Commons Attribution License, which permits unrestricted use, distribution, and reproduction in any medium, provided the original author and source are credited. 
with a rapid increase in local hospital admissions due to Influenza $\mathrm{A}(\mathrm{H} 1 \mathrm{~N} 1) \mathrm{pdm} 09$, which peaked three weeks later (week 46).

In this report, we studied adult patients ( $>15$ years old) admitted to Haukeland University Hospital with confirmed Influenza A(H1N1) pdm09 disease from August 2009 to January 2010. Our aim was to describe the epidemiological characteristics, clinical features, treatment and outcome of these patients. The study period coincided with mass vaccination of both frontline HCWs at the hospital and patient groups at risk in the community, providing a unique situation to study the impact of the vaccine on the course of the pandemic. We hypothesize that early vaccination reduced the burden of the pandemic and protected the integrity of our healthcare system. The continued presence and lack of absenteeism among employees enabled confident and efficient patient care and increased handling capacity. In addition, immunization reduced the need for hospitalization in patients in the community at increased risk of severe influenza disease, thus reducing the burden of mass hospitalization.

\section{Material and Methods}

\section{Study participants}

Adult patients admitted with influenza-like illness (ILI) and hospitalized for more than 24 hours at the Medical and Thoracic Departments, $\mathrm{HUH}$, were eligible to be included in the study. Of the 233 eligible patients, 104 were excluded due to lack of traceability, death from non-related illness, failing to provide informed consent, or not meeting the case definition.

A clinical case definition for Influenza A(H1N1)pdm09 disease (modified CDC case definition) included: temperature $>38^{\circ} \mathrm{C}$ and one of the following criteria; I: ILI symptoms (two of the following symptoms): dry cough, sore throat, runny nose, muscle pain, joint pain, headache, malaise, dyspnea, vomiting/diarrhea; II: Pneumonia; III: Organ failure or collapse. Laboratory confirmed Influenza was defined as a positive (H1N1)pdm09 rt-PCR and/or positive serological antibody titers. All patients provided written, informed consent before inclusion and The Regional Ethical Committee of Western-Norway approved the study.

\section{Study design}

Vaccine: The arrival of the oil-in-water adjuvanated Influenza A (H1N1)pdm09 vaccine (Pandemrix ${ }^{\circledR}$ GlaxoSmithKline, Belgium) to Norway coincided with the increase in Influenza A(H1N1)pdm09 infection rate. Prioritized groups for vaccination were frontline HCWs at the hospital and patients in risk groups in the community. The Centre of Infection Control, HUH organized vaccination of HCWs, and the distribution of vaccine doses was initially strictly limited to frontline HCWs. Mass vaccination of the at risk population and general population was organized by the local health authorities in Bergen municipality. The city's soccer stadium and an elderly center were chosen as venues. Volunteers, retired doctors and nurses performed the vaccination with assistance from the Civil defense. The National Vaccine Register supplied data on the number of vaccinated individuals.

Subjects: Hospitalized patients were asked to provide demographic and clinical information, in addition to access to their medical charts and permission to store blood samples in a bio bank. The data were collected prospectively for each patient, including body-mass index (BMI), smoking and alcohol habits, possible Influenza A(H1N1)pdm09 exposure, influenza vaccination status, co-morbidities, pregnancy, symptoms at onset and upon admission, CRB-65 score at presentation (clinical score predicting mortality in community-acquired pneumonia (scale 0-4)), laboratory and radiological findings including Influenza $\mathrm{A}(\mathrm{H} 1 \mathrm{~N} 1)$ pdm09 rt-PCR and influenza A serology, antiviral and antibiotic treatment, supportive treatment, disease complications and outcome. Acute organ failures were specifically defined [14-16]. Data were collected through interviews with cases, and/or their parent/ guardian or HCW. Physicians and two study nurses performed medical chart abstractions. Each patient was allocated a unique identification number and the data were plotted into SPSS Data entry (version 4.0).

\section{Laboratory methods}

Laboratory confirmation of Influenza $\mathrm{A}(\mathrm{H} 1 \mathrm{~N} 1) \mathrm{pdm} 09$ was performed using swabs taken from the nasopharynx, tonsils, or lower respiratory tract (sterile brush from endotracheal tube or bronchoalveolar lavage) and collected into virus transport medium. All samples were tested at the Department of Microbiology, HUH, using rt-PCR according to CDC (Centre for Disease Control \& Prevention) protocol on a light cycler 2.0 (Roche) [17-19]. Serum samples were collected from some patients and assayed in the complement fixation test (CFT) for influenza A or by haemagglutination inhibition (HI) assay for H1N1pdm09. A positive result was defined as CFT $>32$ or $\mathrm{HI} \geq 40$. Due to the low pre-existing immunity against Influenza A(H1N1)pdm09 in the population, one serum sample with a positive result was considered sufficient for laboratory confirmation of Influenza A(H1N1)pdm09 disease [19-21].

\section{Statistical analysis}

Epidemiological analysis describing the patient population were conducted using PASW (version 18.0), while the graphics were produced using Matlab R2010b. Data quality assurance was undertaken through implementing standard data entry checks and manual checking of entered data against the hard-copy case report forms (CRF). Assessment of risk factors for severe disease was conducted through single variable analysis using the Exact Chi-square test for categorical variables. The general significance level was set to 0.05 . To handle multiple testing effects, we used a Bonferroni adjustment for testing risk factors versus hospital stay, leading to sign level 0.0045 . The length of hospital stay was defined as the difference between discharge date and admission date, and defined as 1 if they were the same day. Severe disease was defined as a hospital stay $>2$ days. The moving median was computed for each day from the median length of hospital stay of all patients admitted during the subsequent 10 days. The moving median was used to highlight time trends.

\section{Results}

\section{The study population}

This study reports on adult patients (aged 15-93 years old, median 44 years old) admitted to $\mathrm{HUH}$ with confirmed Influenza $\mathrm{A}(\mathrm{H} 1 \mathrm{~N} 1)$ pdm09 during the study period. All 129 patients included either met the clinical case definition $(n=119)$ and/or had laboratory confirmed disease $(n=69)$. The majority of patients were $\leq 65$ years old $(81.4 \%)$ and 24 patients $(18.6 \%)$ were $>65$ years old (Table 1 ) The female to male ratio was $\sim 1: 1$ in all age groups, except for 20-50 year olds, where it was 1.6:1.

\section{Vaccination and length of hospital stay}

Mass vaccination of frontline HCWs and patients in risk groups commenced in week 43 (Figure 1). Within two weeks approximately 3000 frontline HCWs at the hospital were vaccinated. In the Infectious Disease Unit, $>95 \%$ of doctors and nurses were immunized by week 45 


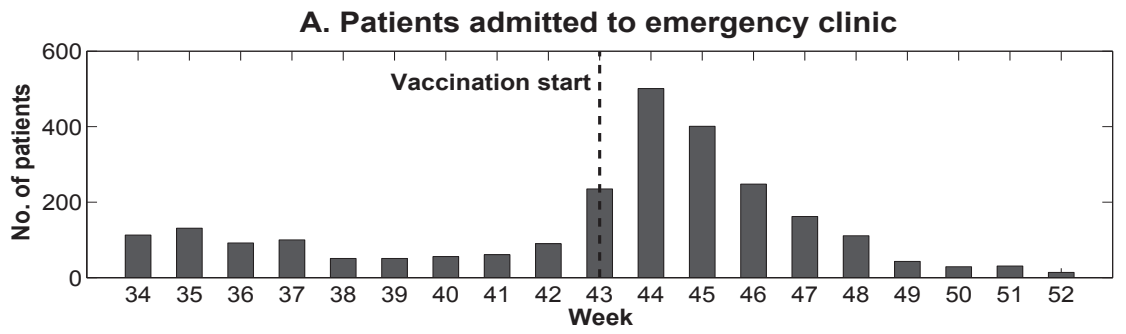

B. Patients admitted to Haukeland University Hospital



C. Vaccinations in Bergen Municipality

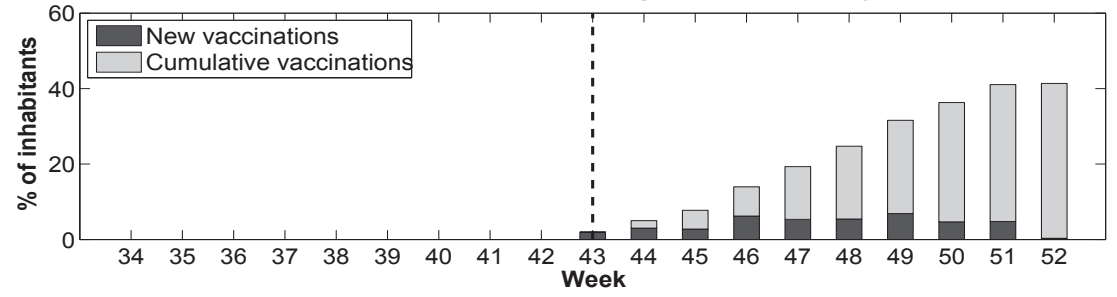

D. Vaccinations of all employees at Haukeland University Hospital

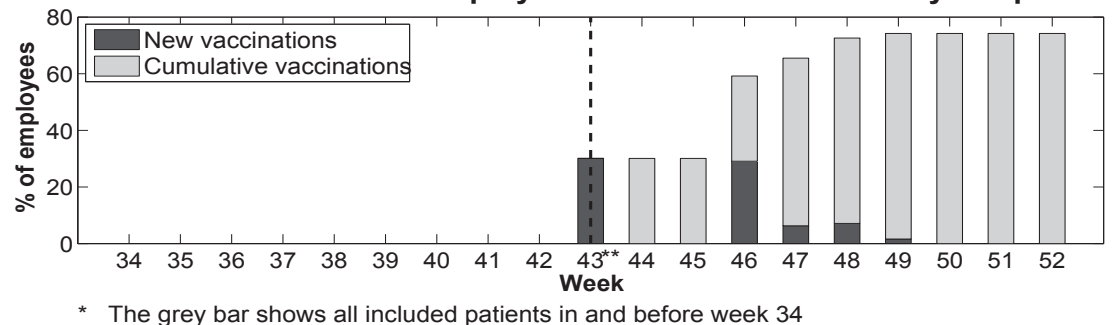

** All vaccines received in week 43 were distributed to frontline health care workers

Figure 1: The time course of the pandemic and the dotted line marks the start of vaccination.

A: Patients consulting the emergency clinic with influenza-like-illness.

$\mathrm{B}$ : The number of patients admitted with suspected H1N1pdm09 to HUH (dark grey bars) and patients included in the study (light grey bars). There are more patients included than admitted in week 34 and 43 , due to patients being recruited from other departments or delayed inclusion.

$\mathrm{C}$ : The number of patients in at risk groups immunized in Bergen. HCWs resident in Bergen are included in the numbers and the figure represents only people < 65 years of age. Light grey bars are the cumulative number of vaccinations; dark grey bars are the number of new vaccinations each week.

$\mathrm{D}$ : The number of health care workers immunized at $\mathrm{HUH}$

(Personal communication, Matron Beate Haaland, Infectious disease Unit, Medical department, HUH). In week 46 the hospital received a second batch of vaccine and vaccination of hospital employees in general continued (Figure 1). In the municipality of Bergen approximately 15 000 people in the WHO defined risk groups were immunized in weeks 43-45, amounting to $1 / 3$ of the estimated 30-40000 people at increased risk of severe disease in Bergen (Personal communication, Senior consultant Dr. Øystein Søbstad, Department of Infection Control, The municipality of Bergen).

Only 25 (19.4\%) of admitted patients had received the pandemic vaccine prior to hospitalization, and 12 patients $(9.5 \%)$ had received the seasonal 2009-2010 inactivated influenza vaccine (Table 1). Despite an increase in number of hospitalizations, there was a significant reduction in length of hospital stay one week after vaccination commenced, in patients admitted with confirmed Influenza $A(\mathrm{H} 1 \mathrm{~N} 1) \mathrm{pdm} 09$ ( $\mathrm{p}=0.029)$ (Figure 2). This reduction was maintained throughout the rest of the study.

\section{Clinical and diagnostic findings}

Symptoms reported upon admission included: fever $>38^{\circ} \mathrm{C}(93 \%)$, cough $(65.9 \%)$, general malaise (58.9\%), vomiting/diarrhea $(38.8 \%)$, dyspnea (37.2\%), and clinically suspected pneumonia (10.9\%) (Table 1). Only one patient presented with organ failure. The median duration of symptoms before hospitalization was 3 days.

Chronic cardiovascular disease (including hypertension) was the most common underlying co-morbidity, followed by chronic pulmonary disease including COPD and immunosuppression 
Citation: Mohn KGI, Lærum BN, Skrede S, Cox RJ, Dyrhol-Riise AM, et al. (2013) Reduced Hospital Stay in Influenza Patients after Mass Vaccination during the 2009 Influenza Pandemic in Norway. J Vaccines Vaccin 4: 197. doi: 10.4172/2157-7560.1000197

Page 4 of 9

\begin{tabular}{|c|c|c|c|c|c|c|}
\hline & \multicolumn{6}{|c|}{$\mathbf{N}$} \\
\hline Total Cases of H1N1 disease & \multicolumn{6}{|c|}{$129(100)$} \\
\hline Clinically confirmed (1) & \multicolumn{6}{|c|}{$119(92.2)$} \\
\hline Lab confirmed ( 2 ) & \multicolumn{6}{|c|}{$70(54.3)$} \\
\hline Confirmed (clinically and lab) & \multicolumn{6}{|c|}{$69(53.5)$} \\
\hline & \multicolumn{2}{|c|}{ Total } & \multicolumn{2}{|r|}{ Female } & \multicolumn{2}{|r|}{ Male } \\
\hline & \multicolumn{2}{|c|}{$\mathrm{N}(\%)$} & \multicolumn{2}{|r|}{$\mathrm{N}(\%)$} & \multicolumn{2}{|r|}{$N(\%)$} \\
\hline Age (median) & \multicolumn{2}{|c|}{$44.1(15.1-93.2)$} & \multicolumn{2}{|c|}{$38.9(15.9-92.8)$} & \multicolumn{2}{|c|}{$51.9(15.1-93.2)$} \\
\hline$<20$ years & 10 & $(7.8) \%$ & 5 & $(7.1) \%$ & 5 & $(8.5) \%$ \\
\hline $20-50$ years & 59 & $(45.7) \%$ & 37 & $(52.9) \%$ & 22 & $(37.3) \%$ \\
\hline $50-65$ years & 36 & $(27.9) \%$ & 18 & $(25.7) \%$ & 18 & $(30.5) \%$ \\
\hline$>65$ years & 24 & $(18.6) \%$ & 10 & $(14.3) \%$ & 14 & $(23.7) \%$ \\
\hline Hospital stay (mean) & 3 range & $(1-125) \%$ & 23.2 & $(15.2-47.7) \%$ & 26.3 & $(18.6-49.2) \%$ \\
\hline BMI (mean) & 24.1 & $(15.2-49.2) \%$ & - & - & - & - \\
\hline Smokers & 33 & $(91.7) \%$ & 17 & $(24.3) \%$ & 16 & $(27.1) \%$ \\
\hline H1N1pdm09-vaccination & 25 & $(19.4) \%$ & 14 & $(20.0) \%$ & 11 & $(18.5) \%$ \\
\hline Seasonal influenza vaccination & 12 & $(9.5) \%$ & 6 & $(9.0) \%$ & 6 & $(10.2) \%$ \\
\hline \multirow{2}{*}{ Clinical features of patients under admission } & \multicolumn{2}{|c|}{ Total } & \multicolumn{2}{|c|}{ Lab confirmed } & Clinically confirmed & Clinically and Lab confirmed \\
\hline & \multicolumn{2}{|c|}{$\mathrm{N}(\%)$} & \multicolumn{2}{|c|}{$N(\%)$} & $N(\%)$ & $N(\%)$ \\
\hline Total & \multicolumn{2}{|c|}{$129(100.0)$} & \multicolumn{2}{|r|}{$70(100.0)$} & $119(100.0)$ & $69(100.0)$ \\
\hline Fever Temperature $>38^{\circ} \mathrm{C}$ & \multicolumn{2}{|c|}{$120(93.0)$} & \multicolumn{2}{|r|}{$61(87.1)$} & $119(100)$ & $60(87.0)$ \\
\hline Cough & \multicolumn{2}{|c|}{$85(65.9)$} & \multicolumn{2}{|r|}{$51(72.9)$} & $79(66.4)$ & $45(65.2)$ \\
\hline General malaise (including headache) & \multicolumn{2}{|c|}{$76(58.9)$} & \multicolumn{2}{|r|}{$40(42.9)$} & $71(59.7)$ & $35(50.7)$ \\
\hline Myalgia & \multicolumn{2}{|c|}{$71(55.0)$} & \multicolumn{2}{|r|}{$38(54.3)$} & $69(58.0)$ & $36(52.2)$ \\
\hline Gastrointestinal symptoms & \multicolumn{2}{|c|}{$50(38.8)$} & & $21(30.0)$ & $48(40.3)$ & $19(27.5)$ \\
\hline Sore throat & & $(38.0)$ & & $26(37.1)$ & $47(39.5)$ & $24(34.8)$ \\
\hline Shortness of breath (dyspnoea) & & $(37.2)$ & & $29(41.4)$ & $45(37.8)$ & $26(37.7)$ \\
\hline Joint aches & & $(23.3)$ & & $19(21.1)$ & $29(24.4)$ & $18(26.1)$ \\
\hline Rhinhorrea & & (19.4) & & $15(21.4)$ & $23(19.3)$ & $13(18.8)$ \\
\hline Clinical pneumonia & & $(10.9)$ & & $6(8.6)$ & $14(11.8)$ & $6(8.7)$ \\
\hline Organ failure & & $(0.8)$ & & $1(1.4)$ & $1(0.8)$ & $1(1.4)$ \\
\hline
\end{tabular}

1. Met the clinical case definition (modified CDC case definition of Influenza A(H1N1)pdm09 disease)

2. Laboratory confirmed cases had positive H1N1pdm09 rt-PCR and/or serology results

Table 1: Demographic and clinical characteristics of hospitalized patients with pandemic Influenza A (H1N1).

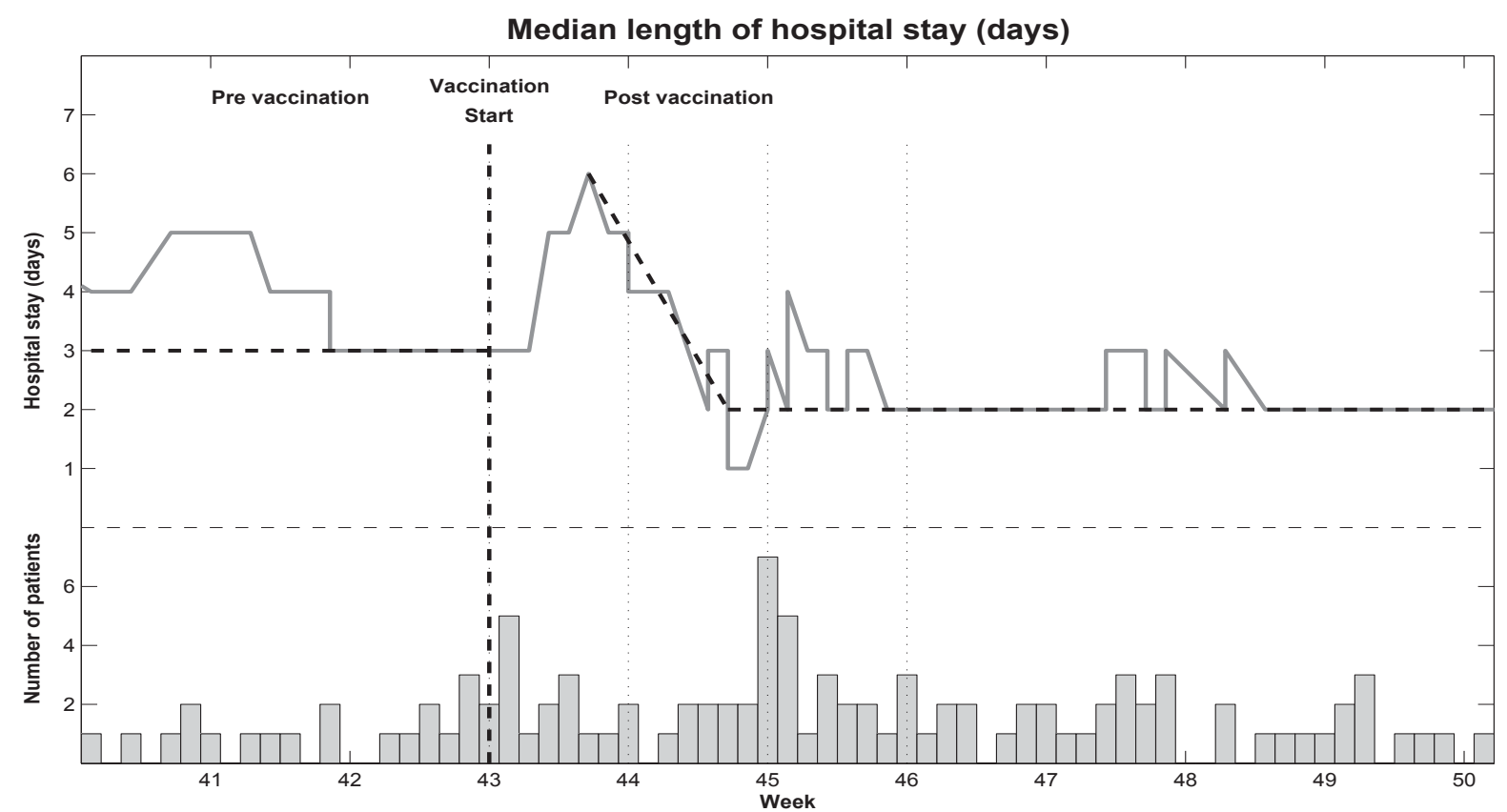

Figure 2: The median length of hospital stay (days) with regard to the timing of mass vaccination.

In the upper part of the figure, the grey line shows the median length of hospital stay of patients admitted during the following 10 days (moving median). The dotted black line illustrates the partial linear trend, showing a significant decrease in median hospital stay (days) starting one week after mass vaccination commenced ( $p=0.029$ ). In the lower part of the figure, the bar chart shows the number of admitted patients included in the study each day. (Not all admitted patients were included in the study). 
Citation: Mohn KGI, Lærum BN, Skrede S, Cox RJ, Dyrhol-Riise AM, et al. (2013) Reduced Hospital Stay in Influenza Patients after Mass Vaccination during the 2009 Influenza Pandemic in Norway. J Vaccines Vaccin 4: 197. doi: 10.4172/2157-7560.1000197

Page 5 of 9

(including HIV infection, bone marrow-or organ transplant) (Table 2). The mean BMI was $24 \mathrm{~kg} / \mathrm{m}^{2}$. All three patients with BMI $>40 \mathrm{~kg} / \mathrm{m}^{2}$, needed intensive care treatment (ICU). Unilateral or bilateral infiltrates were present on chest X-ray in 34 patients (26.6\%) (Table 3). Of the 120 patients with fever $>38^{\circ} \mathrm{C}$, blood cultures were performed in 90 and 11 were positive. The most common bacteria was streptococci. There was no correlation between infiltrate shown on $\mathrm{X}$ - ray and positive bacteriology findings.

H1N1pdm09 rt-PCR was performed on 126 patients, and found positive in 51 patients (40.5\%). Influenza A serology was performed on 47 patients, of whom $32(68.1 \%)$ were positive. Nineteen patients with negative H1N1pdm09 rt -PCR had positive serology results. Two of the three ICU patients had three negative PCR tests before the fourth test, obtained from the lower respiratory tract, was found to be positive.

\section{Treatment and outcome}

The median length of hospital stay was three days (range 1-125 (cut-off)). A length of stay $>2$ days was chosen as a sign of severe disease which was associated with a significantly higher rate of infiltrates on the initial chest $\mathrm{X}$-ray ( 29 vs. $7, \mathrm{p}=0.001)$ and a tendency to more severe hypoxia $(\mathrm{pO} 2<8.0 \mathrm{kPa}$ ) (Figure 3). Patients reporting an underlying medical condition $(n=73)$, specifically, chronic cardiovascular disease or immunosuppression higher risk of increased hospital stay (Figure 3).

\begin{tabular}{|c|c|c|}
\hline \multirow{2}{*}{ Comorbidity } & \multirow{2}{*}{ Data available from $\mathbf{N}$} & Confirmed \\
\hline & & Positive finding $\mathbf{N}(\%)$ \\
\hline No comorbidity & 120 & $47(39.7)$ \\
\hline 1 underlying condition & 120 & $27(22.5)$ \\
\hline 2 underlying conditions & 120 & $22(18.3)$ \\
\hline$>2$ underlying conditions & 120 & $24(20.0)$ \\
\hline Risk factors & & - \\
\hline Chronic cardiovascular disease & 129 & $37(28.7)$ \\
\hline Chronic pulmonary disease & 129 & $35(27.1)$ \\
\hline Asthma & 129 & $15(11.6)$ \\
\hline COPD & 129 & $12(9.3)$ \\
\hline Immunosuppression & 129 & $22(17.1)$ \\
\hline $\mathrm{BMI}>25$ & 120 & $50(41.4)$ \\
\hline $\mathrm{BMI}>30$ & 120 & $16(13.3)$ \\
\hline $\mathrm{BMI}>40$ & 120 & $3(2.5)$ \\
\hline IV drug abuse & 129 & $12(9.3)$ \\
\hline Malignancy & 129 & $9(7.0)$ \\
\hline Chronic liver disease & 129 & $9(7.0)$ \\
\hline Chronic neurological disease & 129 & $9(7.0)$ \\
\hline Diabetes & 129 & $7(5.4)$ \\
\hline Chronic renal disease & 129 & $3(2.3)$ \\
\hline Pregnancy (1) & 70 & $5(7.1)$ \\
\hline
\end{tabular}

(1) Total number of women was 70

Table 2: Co-morbidities and risk factors for severe disease in patients with pandemic influenza A (H1N1)pdm09 disease.

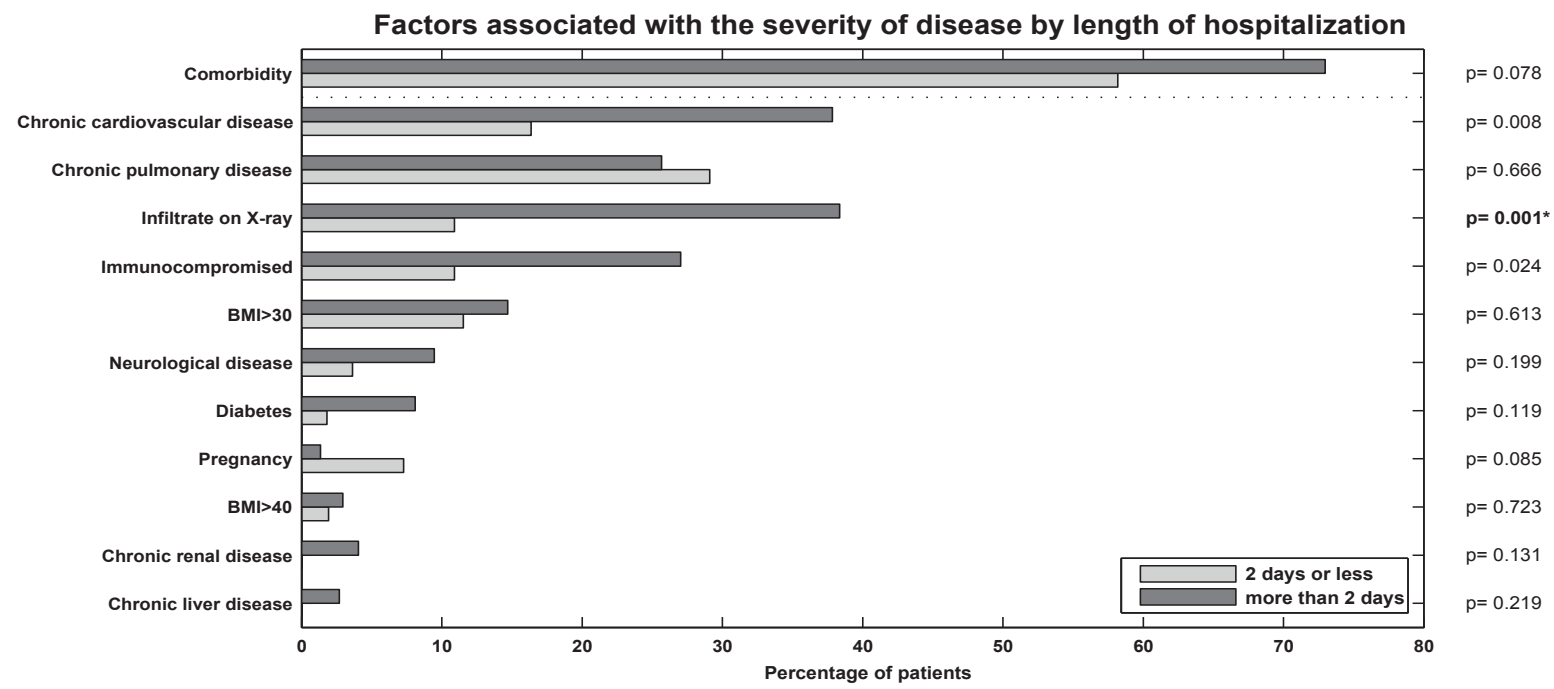

Figure 3: Risk factors associated with disease severity by length of hospital stay. The figure shows the percentage of patients with a defined risk factor divided by the length of hospital stay ( $\leq 2$ days) (light grey bars) and severe illness ( $>2$ days) (dark grey bars). The exact Chi squared test was used to evaluate the association. The $\mathrm{p}$-values reported on the right column are the unadjusted $\mathrm{p}$ values. 
Altogether 95 patients received antiviral treatment (Tamiflu) (73.6\%), with a median time from onset of illness to treatment of 2 days (Table 3). In our cohort $64.4 \%$ of patients received antibiotic treatment, most commonly Penicillin, followed by Cefotaxime. Sixty-three patients (48.8\%) received Oxygen treatment, of which 7 (5.5\%) received noninvasive respiratory support (CPAP/BIPAP). Hypotension requiring vasopressor treatment occurred in $4(3.1 \%)$ patients.

The three patients $(2.1 \%)$ requiring ICU treatment all had fever $>38^{\circ} \mathrm{C}$ upon admission, were severely hypoxic (lowest had pO2 5.10 $\mathrm{kPa}$ ), and therefore put on a ventilator (including oscillator). They were hypotensive, required vasopressor treatment, and had initial CRP counts $>200$. Two of the 3 patients had been infected abroad and none of them were vaccinated. They all had co-morbidities (1): COPD and obesity; (2): asthma, IV drug abuse and BMI $<15 \mathrm{~kg} / \mathrm{m}^{2}$ and (3): cardiovascular risk, diabetes and obesity. They had CRB scores of 1,2 and 3 respectively, thus this was not a predictor of severe disease.

None of the study patients died in hospital. The majority of patients were discharged, recorded as "healthy/getting better/unchanged function in daily life". Fourteen patients were discharged with "increased need of help/support in daily life".

One patient with confirmed Influenza A(H1N1)pdm09 disease and with BMI $47 \mathrm{~kg} / \mathrm{m}^{2}$, survived despite multi organ failure. This patient was hospitalized for 18 months, but followed the study for 125 days, the maximum time frame in this study. The patient was discharged to a rehabilitation center with tetra paralysis as sequelae, but cognitively intact. Despite having COPD and obesity, this patient had chosen not to be vaccinated.

\section{Discussion}

The goal of pandemic vaccination is to elicit appropriate immunological effector mechanisms to reduce viral replication and shedding, thus reduce transmission and provide protection against serious illness and death from influenza. In Bergen, Norway, the pandemic virus spread rapidly from the beginning of November 2009 and hospitalizations peaked three weeks after the onset of mass vaccination. At risk patients in the community and frontline HCWs at the hospital received the first batches of vaccine (Pandemrix ${ }^{\mathbb{E}}$ ) arriving mid October in week 43.
This is a unique report on hospitalized patients with confirmed Influenza $\mathrm{A}(\mathrm{H} 1 \mathrm{~N} 1) \mathrm{pdm} 09$ disease, where mass vaccination of the at risk population in the community and frontline HCWs at the hospital was performed prior to the peak in pandemic activity.

Despite the further increase in the number of hospitalizations, there was a significant reduction in the length of hospital stay in patients admitted with confirmed Influenza $\mathrm{A}(\mathrm{H} 1 \mathrm{~N} 1) \mathrm{pdm} 09$ one week after mass vaccination commenced (Figure 2). This important finding we mainly attribute to the effect of vaccination. We experienced unparalleled immunization coverage of frontline HCWs at the hospital $(>90 \%)$ and $>$ a third of patients at increased risk of severe influenza in the community in weeks $43-45$. We have earlier shown that protective immunity is elicited as early as 6-7 days post vaccination with adjuvant pandemic vaccine [20]. Clinical trials of candidate pandemic avian vaccines have shown 2 doses of an adjuvanted vaccine are needed to provide protective immunity. In contrast only one dose of the pandemic vaccine was required. Although no serological response was detectable in most of the population to the novel H1N1 pandemic virus, vaccines studies revealed that one dose of the adjuvanated pandemic vaccine elicited high antibody titers and hence protection within a week of vaccination. Despite the novel HA, vaccination elicited a rapid secondary response to cross reactive epitopes, hence the vaccine acted as a booster and not a priming dose of vaccine [22].

We hypothesize that the observed reduction in length of hospital stay, is due to a combined effect of lack of absenteeism of staff and reduced mass hospitalization (Figure 2). One man died prior to vaccine availability, but there were no further deaths in hospital after mass vaccination commenced.

The low HCW absenteeism possibly improved clinical confidence among medical staff and increased the ability for quick and safe patient turnover throughout the pandemic. This may also have contributed to the reduced hospital stay. It is there for likely that Norway experienced a reduced burden of the pandemic influenza disease. The burden could have been further eased, had the vaccine arrived just weeks earlier $[23,24]$.

In our cohort, of hospitalized, infected patients, only $19 \%$ were vaccinated (compared to $45 \%$ in the general population), indicating

\begin{tabular}{|c|c|c|c|}
\hline Diagnostic findings & Data available from & Confirmed cases & Positive finding \\
\hline Infiltrate on chest radiography & 128 & 34 & $(26.6)$ \\
\hline Positive H1N1 PCR & 126 & 51 & $(40.5)$ \\
\hline Positive H1N1 serology & 47 & 32 & $(68.1)$ \\
\hline Negative PCR and positive serology & 84 & 19 & $(22.6)$ \\
\hline Positive blood culture & 90 & 9 & $(10)$ \\
\hline CRB score (1) & 90 & 7 & $(85.5)$ \\
\hline $0-1$ & 90 & 7 & $(85.5)$ \\
\hline 2 & 90 & 7 & $(11.1)$ \\
\hline $3-4$ & 90 & 10 & $(3.3)$ \\
\hline CRP count $>1$ & 128 & 41.5 & $(1-593)$ \\
\hline Oxygen saturation at presentation $\left(\% \mathrm{O}_{2}\right)$ & 108 & 97 & $(50-100)$ \\
\hline \multicolumn{4}{|l|}{ Treatment } \\
\hline Oseltavimir (Tamiflu) & 129 & 95 & $(73.6)$ \\
\hline Zanamivir (Relenza) & 129 & 0 & 0 \\
\hline Antibiotic therapy & 128 & 83 & $(64.4)$ \\
\hline Oxygen treatment & 128 & 63 & $(48.8)$ \\
\hline Non-invasive respiratory support & 127 & 4 & $(5.5)$ \\
\hline Vasopressor treatment & 129 & 4 & $(3.1)$ \\
\hline
\end{tabular}

Table 3: Diagnostic findings and clinical treatment of patients with influenza A (H1N1) pdm09. 
that vaccination prevented hospitalization. This is supported by findings from Canada, which found mass vaccination to be cost effective $[25,26]$. Twenty-five patients were vaccinated with Pandemrix ${ }^{\circledR}$ prior to hospital admission of which 11 patients had confirmed Influenza $\mathrm{A}(\mathrm{H} 1 \mathrm{~N} 1) \mathrm{pdm} 09$. This indicates that they probably were infected and vaccinated simultaneously, without suitable time to gain protective immunity. Modeling of the effect of mass vaccination on preventing viral spread in the Norwegian community, suggested that vaccination did not commence in time to strongly influence the pandemic [24]. However, the western part of Norway, where our study was performed, experienced a slightly later wave of pandemic influenza and importantly focuses on patients requiring hospitalization due to influenza infection.

The length of hospital stay may be seen as a parameter of severity of disease as well as the ability of the hospital to rapidly handle the mass influx of patients with milder disease within the hospital's normal capacity. These findings suggest that the pandemic vaccine provided protection from influenza illness, avoiding hospitalization in patients at highest risk of severe disease who would have required longer hospital stay (Figure 2) [5,25-27].

Timely vaccination has been estimated to have significant effect on morbidity, ICU need and mortality $[2,4,25,28-30]$. A study in Scotland concluded that the use of pandemic vaccine was associated with reduction in the burden of consultations with health care providers, emergency hospital admissions and most importantly mortality [30]. Our study and clinical experience supports this modeling. In Bergen, the early deployment of vaccine allowed rapid protection of front line HCWs, reduced absenteeism, despite experiencing substantial pandemic activity, thus allowing the integrity of the health care system to be maintained $[20,25]$. Our hospital did not experience the predicted severe mass hospitalization and avoided setting pandemic emergency plans in action such as cancelling elective surgery. A case control study from nine hospitals in Berlin suggested a protective effect of the pandemic vaccine for the prevention of hospitalization despite low vaccination coverage [31].

There is an on-going discussion in the USA regarding mandatory influenza vaccination of HCWs, and it would be interesting to compare absenteeism and patients' length of hospital stay in institutions that conducted mandatory or voluntary vaccination of HCWs, respectively $[4,5,28,29,32]$.

This study has several limitations. The cohort represented 129 of the 233 adults admitted with suspected pandemic influenza, as we only included patients with confirmed disease, and were obliged to include only patients who provided written informed consent. Some information may therefore have been lost. Some patients may have gone undetected if initially admitted to another ward, or if they did not have fever or respiratory symptoms. In the later stages of the pandemic, the proportion of prospectively included cases decreased due to capacity limitations. Inclusion was performed retrospectively for approximately $25 \%$ of patients. The study was conducted during the heat of the pandemic without the capacity to collect convalescence serological samples. Although the study design was not primarily intended to evaluate the effect of vaccination per se, our study indicates that the Influenza A(H1N1)pdm09 pandemic peaked at a lower level than anticipated, possibly due to mass vaccination.

To lessen the burden on health care services, pharmacists could temporarily prescribe anti-neuraminidase drugs with effect from November 3rd 2009 (week 43). Furthermore, people with suspected pandemic Influenza infection were advised to stay at home for 7 days on paid sick leave, not requiring a doctor's note. Both measures contributed to lower levels of viral shedding in the community, however, the direct impact on reduced disease burden has not been evaluated in this study.

Data from the national Institute of Publlic Health (NIPH) in Norway show that approximately 1300 patients were hospitalized, nearly 200 were treated in ICU and 32 patients died from virologically confirmed Influenza $\mathrm{A}(\mathrm{H} 1 \mathrm{~N} 1)$ pdm09 only 1 of which had been vaccinated [33]. Although potentially lethal, Influenza $A(\mathrm{H} 1 \mathrm{~N} 1)$ pdm09 generally caused mild disease, reflected in the short length of hospital stay in this study population. Patients were often young, otherwise healthy adults, who for a limited period were in need of observation, oxygen, antiviral treatment, and/or antibiotics.

Individuals $\geq 50$ years showed a lower incidence of Influenza A(H1N1)pdm09 infection, probably due to partial pre-existing immunity from previous exposure to H1N1 infection [19,21] The present study included hospitalized adults (mean age $44.1 \mathrm{yrs}$.), adding to the growing body of literature showing a marked lower age distribution for Influenza A(H1N1)pdm09 infected patients than seasonal influenza, where the vast majority of infected patients are $>65$ years. The lower age distribution has been calculated to increase the estimated numbers of years of life lost (YLL) by 3-5 times compared to seasonal influenza, to 9.7 million years. Globally, South East Asia was the region with the highest YLL [34].

The study population consisted of more women than men and thus differs from the nationally reported equal gender distributions in Norway and reports from other countries where male patients dominated suspected Influenza A(H1N1)pdm09 cases [11,35-37]. The difference may be due to Norway having one of the highest percentages worldwide of working women (84\%), hence influencing women to seek medical care when ill, with a female/ male ratio of 4.7:3.0. Furthermore, the female to male ratio in our cohort was almost 1 in all age groups, except for the 20-50 years old where this ratio doubled, reflecting that women are more often the main caretakers of young children, and hence more exposed to infection.

Early reports of the Influenza A(H1N1)pdm09 disease found that obesity was a risk factor for severe disease, though the mechanism remains uncertain $[38,23,39]$. There were a marked lower proportion of obese patients $(13.3 \%)$ in our study than reported elsewhere (32$58 \%$ ) $[7,36,39-41]$, reflected by only $7 \%$ of adults ( $>16$ years) in Norway being defined as obese [7,30,33-35]. However the most obese patient in this study was also the most severely ill, surviving total organ failure.

The clinical findings mirror reports from other countries that fever, dry cough and general malaise were the predominant symptoms upon hospital admission [35,39-43]. Furthermore, the majority of patients had underlying co-morbidities, most commonly cardiovascular disease.

In agreement with other reports, we found that the incidence of laboratory confirmed cases greatly underestimate the impact of the disease [43]. At the peak of the pandemic, rt-PCR test results were delayed due to overwhelming demand and limited laboratory capacity, resulting in the low number of positive PCR tests. The WHO global mortality numbers for the pandemic are based on reported laboratory confirmed pandemic-associated deaths. Less than $12 \%$ were reported to the WHO from the most densely populated regions [34]. Recent modeling has indicated that the WHO estimated global mortality from pandemic Influenza could be underestimated by more than a tenfold (15 times) and 51\% of pandemic deaths estimated in Southeast Asia and Africa [34]. The disproportionate burden of pandemic disease born 
Citation: Mohn KGI, Lærum BN, Skrede S, Cox RJ, Dyrhol-Riise AM, et al. (2013) Reduced Hospital Stay in Influenza Patients after Mass Vaccination during the 2009 Influenza Pandemic in Norway. J Vaccines Vaccin 4: 197. doi: 10.4172/2157-7560.1000197

by Africa and Southeast Asia emphasizes the importance of equitable distribution of future vaccines.

In conclusion, Norway was one of the first European countries to receive the pandemic vaccine, which arrived prior to the peak of the pandemic. This study observed a reduction in hospital stay in patients with confirmed pandemic influenza, whilst experiencing high immunization coverage of frontline HCWs at the hospital and at risk patients in the community. This suggests that mass vaccination of these prioritized groups commenced in time to elicit protection from serious effects of influenza, securing the integrity of our healthcare system.

\section{Acknowledgement}

We wish to thank Marianne Sævik, Marita Wallevik, Turid Helen Felli Lunde, and the staff at the Infectious Disease ward and the Department of internal medicine for help with the study. Furthermore, we thank Consultant Dr Frank van Betten Bergen Emergency ward for providing data on patients with influenza-like illness, and advisor Inger Lise Haugen, Department of Vaccine, Division of Infectious Disease Control, at The Norwegian Institute of Public Health (NIPH) for vaccination data. Haukeland University Hospital, Bergen Clinical Vaccine Consortium and the Influenza Centre at the University of Bergen funded the study intramurally.

\section{References}

1. Sullivan SJ, Jacobson RM, Dowdle WR, Poland GA (2010) 2009 H1N1 influenza. Mayo Clin Proc 85: 64-76.

2. Lagacé-Wiens PR, Rubinstein E, Gumel A (2010) Influenza epidemiology-past, present, and future. Crit Care Med 38: e1-9.

3. Centers for Disease Control Prevention. Outbreak of swine-origin influenza A (H1N1) virus infection - Mexico, March-April 2009. MMWR Morbidity and mortality weekly report 2009; 58(17): 467-70.

4. Poland GA (2010) Mandating influenza vaccination for health care workers: putting patients and professional ethics over personal preference. Vaccine 28: 5757-5759.

5. Strategic Advisory Group of Experts on Immunization - report of the extraordinary meeting on the influenza A (H1N1) 2009 pandemic, 7 July 2009 Wkly Epidemiol Rec 84: 301-304.

6. Releve epidemiologique hebdomadaire / Section d'hygiene du Secretariat de la Societe des Nations $=$ Weekly epidemiological record $/$ Health Section of the Secretariat of the League of Nations 2009; 84(30): 301-4.

7. National Center for I, Respiratory Diseases CDC, Centers for Disease Control Prevention. Use of influenza A (H1N1) 2009 monovalent vaccine: recommendations of the Advisory Committee on Immunization Practices (ACIP), 2009. MMWR Recommendations and reports: Morbidity and mortality weekly report Recommendations and reports / Centers for Disease Control 2009; 58(RR-10): 1-8.

8. Centers for Disease Control and Prevention (CDC) (2009) Update: nove influenza A (H1N1) virus infection - Mexico, March-May, 2009. MMWR Morb Mortal Wkly Rep 58: 585-589.

9. McLean E, Pebody RG, Campbell C, Chamberland M, Hawkins C, et al. (2010) Pandemic (H1N1) 2009 influenza in the UK: clinical and epidemiological findings from the first few hundred (FF100) cases. Epidemiol Infect 138: 1531-1541.

10. Perez-Padilla R, de la Rosa-Zamboni D, Ponce de Leon S, Hernandez M Quiñones-Falconi $F$, et al. (2009) Pneumonia and respiratory failure from swine-origin influenza A (H1N1) in Mexico. N Engl J Med 361: 680-689.

11. Rodriguez A, Socias L, Guerrero JE (2010) Pandemic influenza A in the ICU: experience in Spain and Latin America. GETGAG/SEMICYUC/(Spanish Working Group on Severe Pandemic Influenza A/SEMICYUC)]. Medicina intensiva / Sociedad Espanola de Medicina Intensiva y Unidades Coronarias 34: 87-94.

12. Domínguez-Cherit G, Lapinsky SE, Macias AE, Pinto R, Espinosa-Perez L, et al. (2009) Critically III patients with 2009 influenza $A(H 1 N 1)$ in Mexico. JAMA 302: 1880-1887.

13. ANZIC Influenza Investigators, Webb SA, Pettilä V, Seppelt I, Bellomo R, et al. (2009) Critical care services and 2009 H1N1 influenza in Australia and New Zealand. N Engl J Med 361: 1925-1934.
14. Srisawat N, Hoste EE, Kellum JA (2010) Modern classification of acute kidney injury. Blood Purif 29: 300-307.

15. Cox NR (2009) Acute Liver Failure. Hospital Physician

16. Dickstein K, Cohen-Solal A, Filippatos G, McMurray JJ, Ponikowski P, et al. (2008) ESC guidelines for the diagnosis and treatment of acute and chronic heart failure 2008: the Task Force for the diagnosis and treatment of acute and chronic heart failure 2008 of the European Society of Cardiology. Developed in collaboration with the Heart Failure Association of the ESC (HFA) and endorsed by the European Society of Intensive Care Medicine (ESICM). Eur J Heart Fail 10: $933-989$.

17. Ward CL, Dempsey MH, Ring CJ, Kempson RE, Zhang L, et al. (2004) Design and performance testing of quantitative real time PCR assays for influenza $A$ and B viral load measurement. J Clin Virol 29: 179-188.

18. Liao RS, Landt O, Hill JT (2011) Comparison of a laboratory-developed RTPCR and the CDC RT-PCR protocol with rapid immunodiagnostic testing during the 2009 H1N1 influenza A pandemic. Diagn Microbiol Infect Dis 70: 236-239.

19. Hancock K, Veguilla V, Lu X, Zhong W, Butler EN, et al. (2009) Cross-reactive antibody responses to the 2009 pandemic $\mathrm{H} 1 \mathrm{~N} 1$ influenza virus. N Engl J Med 361: 1945-1952.

20. Madhun AS, Akselsen PE, Sjursen H, Pedersen G, Svindland S, et al. (2010) An adjuvanted pandemic influenza $\mathrm{H} 1 \mathrm{~N} 1$ vaccine provides early and long term protection in health care workers. Vaccine 29: 266-273.

21. Miller E, Hoschler K, Hardelid P, Stanford E, Andrews N, et al. (2010) Incidence of 2009 pandemic influenza A H1N1 infection in England: a cross-sectional serological study. Lancet 375: 1100-1108.

22. Li GM, Chiu C, Wrammert J, McCausland M, Andrews SF, et al. (2012) Pandemic $\mathrm{H} 1 \mathrm{~N} 1$ influenza vaccine induces a recall response in humans that favors broadly cross-reactive memory B cells. Proc Natl Acad Sci U S A 109: 9047-9052.

23. Morgan OW, Bramley A, Fowlkes A, Freedman DS, Taylor TH, et al. (2010) Morbid obesity as a risk factor for hospitalization and death due to 2009 pandemic influenza A(H1N1) disease. PLoS One 5: e9694.

24. Blasio BF, Iversen BG, Tomba GS (2012) Effect of vaccines and antivirals during the major $2009 \mathrm{~A}(\mathrm{H} 1 \mathrm{~N} 1)$ pandemic wave in Norway--and the influence of vaccination timing. PLoS One 7: e30018.

25. Sander B, Bauch CT, Fisman D, Fowler RA, Kwong JC, et al. (2010) Is a mass immunization program for pandemic (H1N1) 2009 good value for money? Evidence from the Canadian Experience. Vaccine 28: 6210-6220.

26. Puig-Barberà J, Arnedo-Pena A, Pardo-Serrano F, Tirado-Balaguer MD, PérezVilar S, et al. (2010) Effectiveness of seasonal 2008-2009, 2009-2010 and pandemic vaccines, to prevent influenza hospitalizations during the autumn 2009 influenza pandemic wave in Castellon, Spain. A test-negative, hospitalbased, case-control study. Vaccine 28: 7460-7467.

27. Bone A, Guthmann JP, Nicolau J, Lévy-Bruhl D (2010) Population and risk group uptake of $\mathrm{H} 1 \mathrm{~N} 1$ influenza vaccine in mainland France 2009-2010: results of a national vaccination campaign. Vaccine 28: 8157-8161.

28. Poland GA (2010) The 2009-2010 influenza pandemic: effects on pandemic and seasonal vaccine uptake and lessons learned for seasonal vaccination campaigns. Vaccine 28 Suppl 4: D3-13.

29. Keller DM (2013) Hospital reaches near $100 \%$ flu vaccination of staff with mandate.

30. Simpson CR, Ritchie LD, Robertson C, Sheikh A, McMenamin J (2012) Effectiveness of $\mathrm{H} 1 \mathrm{~N} 1$ vaccine for the prevention of pandemic influenza in Scotland, UK: a retrospective observational cohort study. Lancet Infect Dis 12 : 696-702.

31. Hellenbrand W, Jorgensen P, Schweiger B, Falkenhorst G, Nachtnebel M et al. (2012) Prospective hospital-based case-control study to assess the effectiveness of pandemic influenza $\mathrm{A}(\mathrm{H} 1 \mathrm{~N} 1)$ pdm09 vaccination and risk factors for hospitalization in 2009-2010 using matched hospital and testnegative controls. BMC Infect Dis 12: 127.

32. Salgado CD, Giannetta ET, Hayden FG, Farr BM (2004) Preventing nosocomial influenza by improving the vaccine acceptance rate of clinicians. Infect Control Hosp Epidemiol 25: 923-928.

33. Aavitsland Preben F (2009) Rapport om dødsfall av influensa $A(H 1 N 1)$. 
Citation: Mohn KGI, Lærum BN, Skrede S, Cox RJ, Dyrhol-Riise AM, et al. (2013) Reduced Hospital Stay in Influenza Patients after Mass Vaccination during the 2009 Influenza Pandemic in Norway. J Vaccines Vaccin 4: 197. doi: 10.4172/2157-7560.1000197

34. Dawood FS, luliano AD, Reed C, Meltzer MI, Shay DK, et al. (2012) Estimated global mortality associated with the first 12 months of 2009 pandemic influenza A H1N1 virus circulation: a modelling study. Lancet Infect Dis 12: 687-695.

35. Donker T, van Boven M, van Ballegooijen WM, Van't Klooster TM, Wielders CC et al. (2011) Nowcasting pandemic influenza A/H1N1 2009 hospitalizations in the Netherlands. Eur J Epidemiol 26: 195-201.

36. Cui W, Zhao H, Lu X, Wen Y, Zhou Y, et al. (2010) Factors associated with death in hospitalized pneumonia patients with 2009 H1N1 influenza in Shenyang, China. BMC Infect Dis 10: 145.

37. Gómez-Gómez A, Magaña-Aquino M, Garcia-Sepúlveda C, Ochoa-Pérez UR, Falcón-Escobedo R, et al. (2010) Severe pneumonia associated with pandemic (H1N1) 2009 outbreak, San Luis Potosí, Mexico. Emerg Infect Dis 16: 27-34.

38. Louie JK, Acosta M, Samuel MC, Schechter R, Vugia DJ, et al. (2011) A novel risk factor for a novel virus: obesity and 2009 pandemic influenza $A(H 1 N 1)$. Clin Infect Dis 52: 301-312.
39. Kumar A, Zarychanski R, Pinto R, Cook DJ, Marshall J, et al. (2009) Critically ill patients with 2009 influenza A(H1N1) infection in Canada. JAMA 302: 1872-1879.

40. Lee EH, Wu C, Lee EU, Stoute A, Hanson H, et al. (2010) Fatalities associated with the 2009 H1N1 influenza A virus in New York city. Clin Infect Dis 50: 1498-1504.

41. Jain S, Kamimoto L, Bramley AM, Schmitz AM, Benoit SR, et al. (2009) Hospitalized patients with $2009 \mathrm{H} 1 \mathrm{~N} 1$ influenza in the United States, April-June 2009. N Engl J Med 361: 1935-1944.

42. Louie JK, Acosta M, Winter K, Jean C, Gavali S, et al. (2009) Factors associated with death or hospitalization due to pandemic 2009 influenza $A(H 1 N 1)$ infection in California. JAMA 302: 1896-1902.

43. Brandsaeter BJ, Pillgram M, Berild D, Kjekshus H, Kran AM, et al. (2011) Hospitalised patients with suspected $2009 \mathrm{H} 1 \mathrm{~N} 1$ influenza $A$ in a hospital in Norway, July - December 2009. BMC Infect Dis 11: 75. 